\title{
SAR in human head model due to resonant wireless power transfer system
}

\author{
Chao Zhang ${ }^{\mathrm{a}, \mathrm{b}}$, Guoqiang Liu ${ }^{\mathrm{a}, *}$, Yanhong Li ${ }^{\mathrm{a}, \mathrm{b}}$ and Xianjin Song ${ }^{\mathrm{a}, \mathrm{b}}$ \\ ${ }^{a}$ Institute of Electrical Engineering, Chinese Academy of Sciences, Beijing, China \\ ${ }^{\mathrm{b}}$ University of Chinese Academy of Sciences, Beijing, China
}

\begin{abstract}
.
BACKGROUND: Efficient mid-range wireless power transfer between transmitter and the receiver has been achieved based on the magnetic resonant coupling method. The influence of electromagnetic field on the human body due to resonant wireless power transfer system (RWPT) should be taken into account during the design process of the system.

OBJECTIVE: To analyze the transfer performance of the RWPT system and the change rules of the specific absorption rate (SAR) in the human head model due to the RWPT system.

METHODS: The circuit-field coupling method for a RWPT system with consideration of the displacement current was presented. The relationship between the spiral coil parameters and transfer performance was studied. The SAR in the human head model was calculated under two different exposure conditions.

RESULTS: A system with output power higher than $10 \mathrm{~W}$ at $0.2 \mathrm{~m}$ distance operating at a frequency of approximately $1 \mathrm{MHz}$ was designed. The FEM simulation results show the peak SAR value is below the safety limit which appeared when the human head model is in front of the transmitter.

CONCLUSION: The simulation results agreed well with the experimental results, which verified the validity of the analysis and design.
\end{abstract}

Keywords: Wireless power transfer, SAR, resonant coupling, field-circuit coupling method, FEM

\section{Introduction}

Recently, high efficient mid-range wireless power transfer between transmitter and receiver has been achieved based on magnetic resonant coupling method [1,2]. Numerous applications can be found in the medical field [3], including deep brain stimulator, pacemakers, cochlear hearing implants and other health monitor devices. However, the strong electromagnetic fields of RWPT system may induce high electromagnetic fields in the human body in their closest vicinity. It is necessary to take into account the human safety during such system design process because the transfer power is much larger than that in wireless communication [4].

The basic restrictions for electromagnetic fields in terms of the specific absorption rate (SAR) in the body are provided in the Safety guidelines for electromagnetic field exposure of both workers and general public issued by the International Commission Non-Ionizing Radiation Protection and the Institute of Electrical and Electronics Engineers to prevent damage to the human body's tissues [5,6]. According

\footnotetext{
${ }^{*}$ Corresponding author: Guoqiang Liu, Institute of Electrical Engineering, Chinese Academy of Sciences, Beijing 100190, China. Tel.: +86 10 82547190; Fax: +86 10 82547189; E-mail: gqliu@ mail.iee.ac.cn.
}

0928-7329/16/\$35.00 (C) 2016 - IOS Press and the authors. All rights reserved This article is published online with Open Access and distributed under the terms of the Creative Commons Attribution NonCommercial License. 


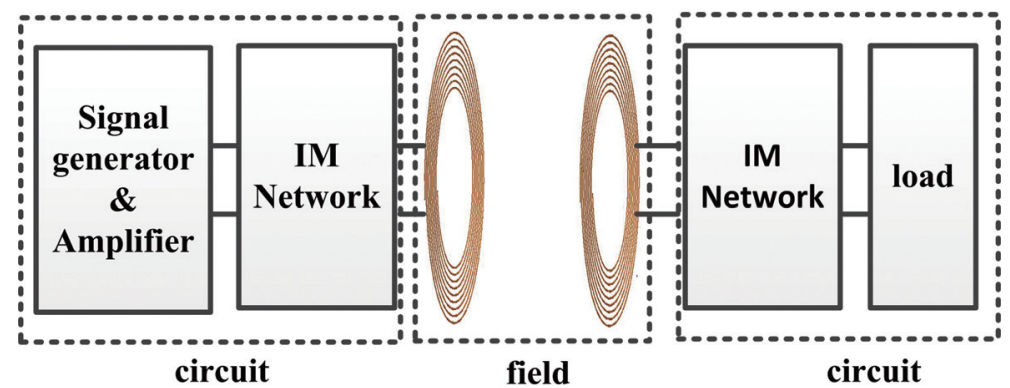

Fig. 1. Schematic of RWPT system.

to it, for the general public, a whole-body averaged SAR is limited of $0.08 \mathrm{~W} / \mathrm{kg}$ and a localized SAR is limited of $2 \mathrm{~W} / \mathrm{kg}$ averaged over $10 \mathrm{~g}$ of tissue.

The study on human exposure to electromagnetic fields from wireless power transfer system is getting much concern in recent years [7,8]. Hirata presented a two-step computational finite difference time domain (FDTD) method to calculate the induced electromagnetic fields in the human body due to the RWPT system at $10 \mathrm{MHz}$ [9]. Christ proposed the total-field scattered-field (TFSF) method to investigate the exposure of a person in the strong coupled near-field of a RWPT system [10].

Considering human exposure to electromagnetic fields from a wireless power transfer system, this paper used Field-circuit coupling method to evaluate the SAR in the human head model induced by RWPT system. The second part is devoted to a brief description of the proposed RWPT system and the circuit-field coupling theory. The coil and matching capacitor selection method and the transfer performance of RWPT system is also put forward. The third part focused on investigating the SAR values in the human head model induced by the designed system under two different conditions. The experiment at $1 \mathrm{MHz}$ frequency is carried out to validate the field-circuit coupling method in the fourth part.

\section{Wireless power transfer system}

\subsection{System structure}

Transmitter and receiver with high quality factor in the wireless power transfer system can efficiently achieve energy transfer over mid-range distances. The designed RWPT system in this paper mainly consists of high frequency signal generator, transmitter and corresponding impedance matching (IM) network, receiver and corresponding IM network and load, as shown in Fig. 1. The transmitter is a spiral coil which is made of copper, and the receiver is the same.

\subsection{Fundamental equations}

The equivalent system is implemented in COMSOL Multiphysics FEM software for simulations. The transfer performance of the system was studied by presented, analyzed and compared Experimental and simulation results.

The relationship between the electric and magnetic field coupled in the time-varying electromagnetic field is described by the Maxwell equations. The exciting source of magnetic field includes conduction 
current and displacement current. Ampere's law in frequency domain included displacement current is as follows:

$$
\nabla \times \boldsymbol{H}=\boldsymbol{J}_{s}+\sigma \boldsymbol{E}+i \omega \varepsilon \boldsymbol{E}
$$

Where, $\boldsymbol{J}_{s}$ is the externally generated current density, $\sigma$ is the conductivity, $\varepsilon$ is the permittivity, $\boldsymbol{E}$ is the electric field intensity, $\boldsymbol{H}$ is the magnetic field intensity, $\sigma E$ is the conduction current density and $i \omega \varepsilon \boldsymbol{E}$ is the displacement current density.

According to the law of magnetic flux continuity, the Gauss theorem and Faraday's law of electromagnetic induction given as follows:

$$
\begin{aligned}
& \nabla \cdot \boldsymbol{B}=0 \\
& \nabla \cdot(\varepsilon \boldsymbol{E})=\rho \\
& \nabla \times \boldsymbol{E}=-i \omega \boldsymbol{B}
\end{aligned}
$$

Where, $\rho$ is the charge density.

It is possible to rewrite Maxwell's equations using the definitions of the magnetic vector potential $A$ and electric scalar potential $u$.

$$
\begin{aligned}
& \boldsymbol{B}=\nabla \times \boldsymbol{A} \\
& \boldsymbol{E}=-\nabla u-i \omega \boldsymbol{A}
\end{aligned}
$$

Thus the equations with $A$ and $u$ are as follows:

$$
\begin{aligned}
& \nabla \times \nabla \times \boldsymbol{A}+\left(i \omega \sigma-\omega^{2} \varepsilon^{2}\right) \boldsymbol{A}+(\sigma+i \omega \varepsilon) \nabla u=-\mu \boldsymbol{J}_{s} \\
& i \omega \nabla \cdot \boldsymbol{A}+\nabla^{2} u=-\rho / \varepsilon
\end{aligned}
$$

The magnetic vector potential is not unique if the divergence of magnetic vector potential were not defined, so it's necessary to add a gauge.

$$
\nabla \cdot \boldsymbol{A}=-\mu \varepsilon \frac{\partial u}{\partial t}
$$

The electric scalar potential is zero on the infinite boundary

$$
u=0
$$

The magnetic insulation boundary condition is satisfied on the infinite boundary

$$
\boldsymbol{n} \times \boldsymbol{A}=0
$$

The transmitter is excited by the primary electric circuit which consists of a voltage source, the primary resistance and the matching capacitor in serious with the transmitting coil. The receiving coil is connected with the secondary electric circuit which consists of the load and the matching capacitor in serious with the transmitting coil, as shown in Fig. 1. 


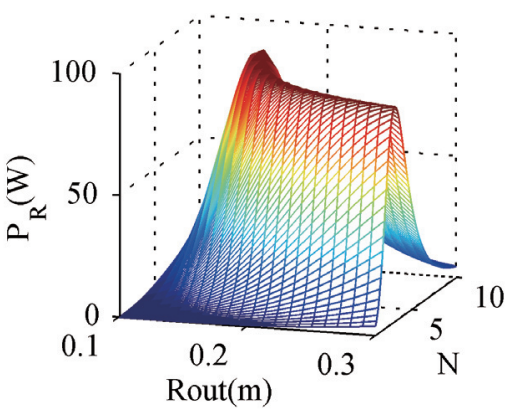

(a)

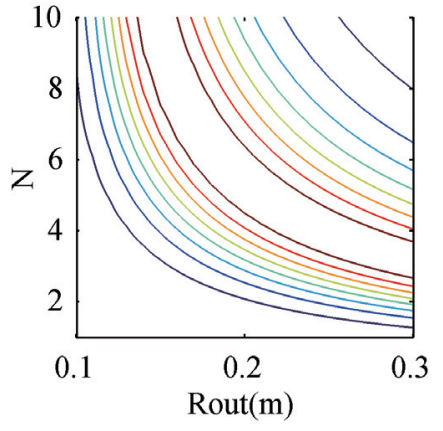

(b)

Fig. 2. The relationship between coil parameter and output power.

The inductance of the coil is calculated from the integral of the magnetic energy density $W_{m}$.

$$
L=\frac{2}{I^{2}} \int_{\Omega} W_{m} d \Omega
$$

Recently the study on transfer performance of RWPT is mainly based on the magnetic quasi-static approximation method which is the fundamental theory of electric current. Only when the displacement current is far smaller than the conduction current does the magnetic field and electric field is decoupled and the quasi-static approximation is valid. Then, the inductance can be calculated with the empirical formula [11].

$$
L=\mu N^{2} R\left[\ln (2.46 / p)+0.2 p^{2}\right]
$$

Where, $\mu$ is the magnetic permeability, $p$ is the fill ratio calculated as $p=\left(R_{\text {out }}-R_{\text {in }}\right) /\left(R_{\text {out }}+R_{\text {in }}\right)$, $R$ is the average radius calculated as $R=\left(R_{\text {out }}+R_{\text {in }}\right) / 2, N$ is the turn of the coil.

\subsection{Coil parameter}

The selection of coil parameters is important for high efficient power transfer between two resonators. In this paper, with the given output power and transfer distance, the spiral coil are wanted to be of high efficiency and less electromagnetic radiation.

Herein given the frequency of the power supply is about $1 \mathrm{MHz}$, the distance between the transmitter and the receiver is $0.2 \mathrm{~m}$, the distance between the coil turns is $4 \mathrm{~mm}$, the radius of the wire is $1 \mathrm{~mm}$ and the load is $400 \mathrm{ohm}$. The output power as a function of the coil outer radius and the coil turns is shown in Fig. 2(a). Figure 2(b) shows the projection when the output power is higher than $10 \mathrm{~W}$. The value of coil radius and turn number within the shaded area meets the power requirement.

The influence of magnetic field on human body should be regarded during the design process to ensure the safety. The magnetic field intensity is directly influenced by the current in the coil, so the current in the coil is a crucial factor to be considered. The current should not be too large from the view of security and stability. The current in transmitting coil as a function of the coil outer radius and the coil turns is shown in Fig. 3. When the coil turn is fixed, the current will decrease with the increase of the coil radius. When the coil radius is fixed, the current will decrease with the increase of coil turn. Considering both the output power and transmitting current, the turn number of the spiral coil designed in this paper would be nine and the outer radius would be $0.25 \mathrm{~m}$. 


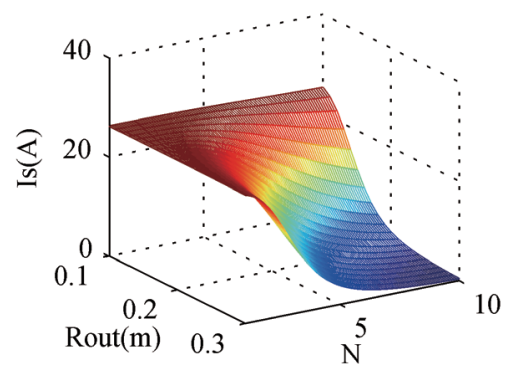

Fig. 3. The current in transmitte.

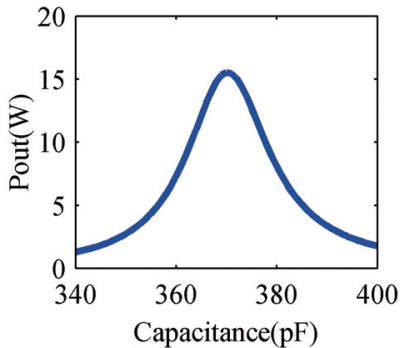

(a)

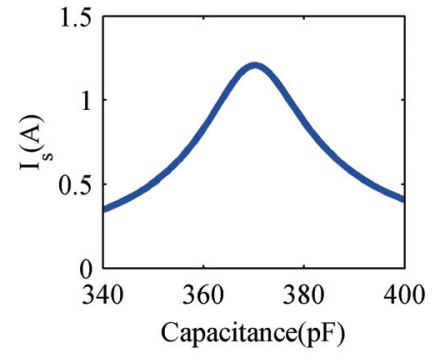

(b)

Fig. 4. The relationship between transfer performance and matching capacitance.

\subsection{Effect of matching capacitor}

The power transfer performance at $1 \mathrm{MHz}$ frequency without the human model closed to the system is shown in Fig. 4. The relationship between output power and matching capacitance is shown in Fig. 4(a), the relationship between transmitting coil current and matching capacitance is shown in Fig. 4(b).

For the capacitance varying from $340 p F$ to $400 p F$, when the capacitance is between $365 p F$ and $380 \mathrm{pF}$, the output power is higher than $10 \mathrm{~W}$ and the transfer efficiency is higher than $80 \%$. The maximum value appears when the matching capacitance is about $370 \mathrm{pF}$. The magnetic field intensity induced by the large current may exceed the human exposure limits and have negative effects on human body. So the current should be as small as possible on the premise that the output power and transfer efficiency meet the needs at the given distance. The matching capacitor selected in this paper is $366 p F$.

\section{Simulation of SAR in head model}

\subsection{Simulation conditions}

With rapid development of the wireless power transfer system, it is particularly important to reduce the radiation into the human body in the vicinity. The SAR value is used to evaluate an average amount of energy absorbed by human beings over a region of $10 \mathrm{~g}$ of tissue.

$$
E_{S A R}=\sigma_{\text {brain }} \frac{|E|^{2}}{\rho_{\text {brain }}}
$$




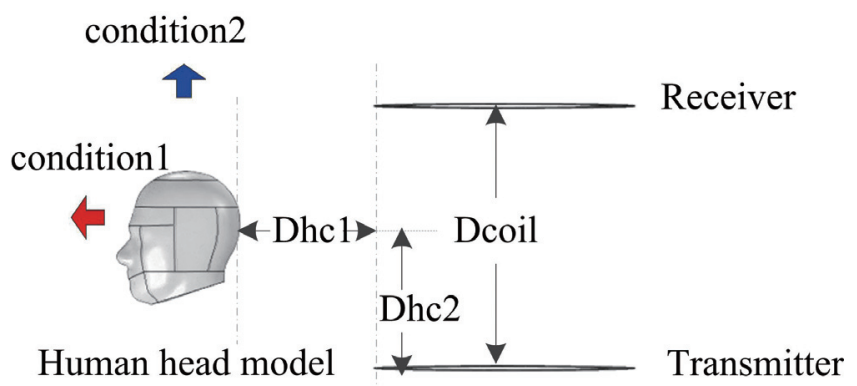

Fig. 5. Geometry of the exposure condition.

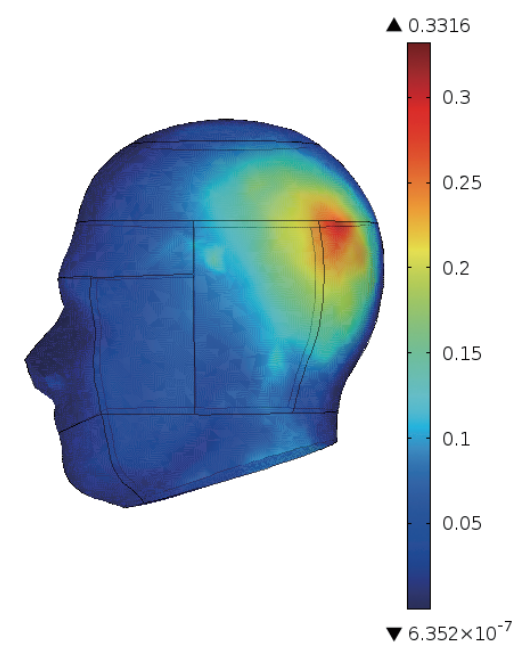

Fig. 6. $10 \mathrm{~g}$ SAR of head model.

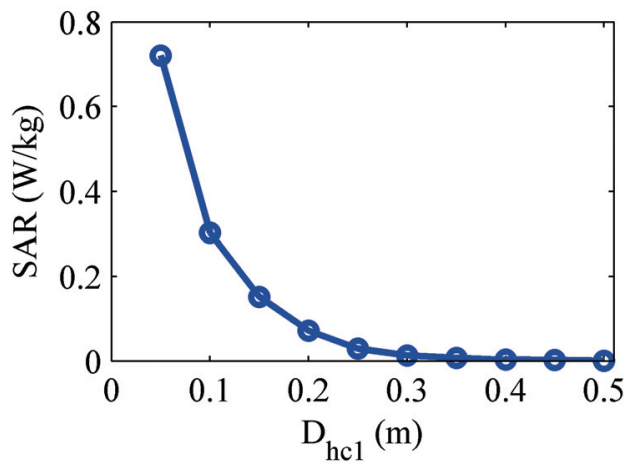

Fig. 7. Peak SAR value when $D_{h c 1}$ is varied.

Where, $\sigma_{\text {brain }}$ is the conductivity of human brain tissue, $\rho_{\text {brain }}$ is the density of human tissue and $|E|$ is the RMS value of the electric field intensity.

The SAR in the human head model closed to the designed RWPT system is calculated in this study. The human head model is as the same as the model provided by IEEE, IEC, and CENELEC from their standard specification of SAR value measurements.

The SAR values under two exposure conditions shown in Fig. 5 are calculated. The two resonant coils are coaxial. The distance between transmitter and receiver represented by $D_{\text {coil }}$ is $0.2 \mathrm{~m}$. The vertical distance between the edge of the human head model and the edge of coil is expressed by $D_{h c 1}$ and the vertical distance between the middle cross section of the head model and the horizontal plane of transmitter is represented by $D_{h c 2}$. Under the first condition, $D_{h c 2}$ is $0.1 \mathrm{~m}$ and $D_{h c 1}$ is varied from 5 $\mathrm{cm}$ to $50 \mathrm{~cm}$. Under the second condition, $D_{h c 1}$ is $0.1 \mathrm{~m}$ and $D_{h c 2}$ is varied from 0 to $D_{\text {coil }}$, i.e., from the transmitter to the receiver.

\subsection{Simulation results}

The peak SAR value of the human head model under the first condition is shown in Fig. 7. When $D_{h c 1}$ is $5 \mathrm{~cm}$, the peak SAR reaches the maximum value $0.72 \mathrm{~W} / \mathrm{kg}$, which is within the specified limit 


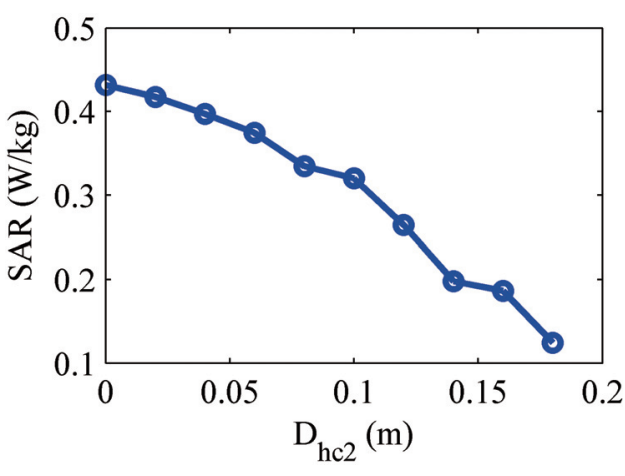

Fig. 8. Peak SAR value when $D_{h c 2}$ is varied.

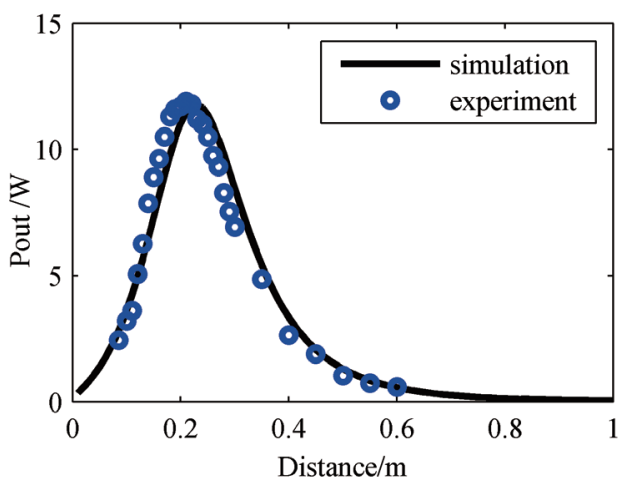

Fig. 9. Experiment and simulation results.

$2 \mathrm{~W} / \mathrm{kg}$. The SAR value would decrease significantly when the head model is moving far away from the RWPT system. The peak SAR value of the human head model under the second condition is shown in Fig. 8. When $D_{h c 2}$ is 0 where the head model is nearest to the transmitter, the peak SAR reaches the maximum value $0.43 \mathrm{~W} / \mathrm{kg}$, which is also within the limit $2 \mathrm{~W} / \mathrm{kg}$. The peak SAR value will be higher when the distance between head model and transmitter becomes closer. The SAR value is highest when the surface of the head facing the incident electromagnetic field, as shown in Fig. 6. The simulation results demonstrate that the RWPT system designed in this paper meets the demand of safety.

\section{Experiment validation}

The experiment was carried out to validate the field-circuit coupling method. A class E amplifier was used to generate electric power at about $1 \mathrm{MHz}$. The system operated under resonant condition by fine tuning the capacitors connected in serious with the coils. The output power is measured with the capacitance is $360 \mathrm{pF}$ and the resistive load is $400 \mathrm{ohm}$. The output power compares with the simulation results are given on the curves in Fig. 9.

The coil inductance calculated by the full-wave field-circuit simulation method and magnetic quasistatic approximation method is $68.1 \mu \mathrm{H}$ and $68.4 \mu \mathrm{H}$ respectively. The inductance measured in the experiment is $68.3 \mu \mathrm{H}$. The experiment leads to coherent results with the simulation. Therefore, the field-circuit coupling method is feasible to simulate the performance of the RWPT system.

\section{Conclusion}

During the design process of RWPT system, the requirements of the transfer performance are satisfied while also the human safety should be taken into account. The relationship between spiral coil parameters and transfer performance is analyzed by the circuit-field coupling method. The power transfer efficiency of the designed system is higher than $80 \%$ when the transfer distance is within $20 \mathrm{~cm}$ at the frequency of about $1 \mathrm{MHz}$. The $10 \mathrm{~g}$ SAR value is calculated with the changing of the relative position of the human head model and the coils. The results show that the exposure is strongly dependent on the distance between the head model and coils. The maximum peak SAR value appears when the head model is close to the transmitter. Moreover, with the increase of distance between the human head model 
and the RWPT system, the peak local SAR value decreases rapidly. The maximum peak SAR value under the given conditions is $0.72 \mathrm{~W} / \mathrm{kg}$, which is lower than the limit $2 \mathrm{~W} / \mathrm{kg}$. The transfer performance is analyzed, and the feasibility of the proposed method was confirmed with experimental results.

\section{Acknowledgements}

This work was supported by the National Natural Science Foundation of China under Grants 51207150, 51137004, 51307007 and 51307164.

\section{References}

[1] A. Karalis, J. Joannopoulos, and M. Soljacic. Efficient wireless non radiative mid-range energy transfer[J]. Annals of Physics. 2008; 323(1): 34-48.

[2] A. Kurs, A. Karalis, R. Moffatt, J.D. Joannopoulos, P. Fisher, M. Soljacic. Wireless power transfer via strongly coupled magnetic resonances[J]. Science. 2007; 317(5834): 83-86.

[3] Park S W, Kim H L, Cho J, et al. Wireless power transmission characteristics for implantable devices inside a human body[C]. Electromagnetic Compatibility, 2014 International Symposium on IEEE. 2014: 1190-1194.

[4] Schiavoni A, Bertotto P, Richiardi G, et al. SAR generated by commercial cellular phones-phone modeling, head modeling, and measurements[J]. IEEE Transactions on Microwave Theory and Techniques. 2000; 48(11): 2064-2071.

[5] ICNIRP. Guidelines for limiting exposure to time-varying electric and magnetic fields (1 Hz to $100 \mathrm{kHz}$ ). Health Phys. 2010: 818-836.

[6] IEEE International Committee on Electromagnetic Safety. IEEE standard for military workplaces-force health protection regarding personnel exposure to electric, magnetic, and electromagnetic Fields, $0 \mathrm{~Hz}$ to $300 \mathrm{GHz}$. 2014: 1-80.

[7] Sunohara T, Laakso I, Chan K H, et al. Compliance of induced quantities in human model for wireless power transfer system at $10 \mathrm{MHz}[\mathrm{C}] / /$ Electromagnetic Theory (EMTS), Proceedings of 2013 URSI International Symposium on IEEE. 2013: 831-833.

[8] Sunohara T, Laakso I, Hirata A, et al. Induced field and SAR in human body model due to wireless power transfer system with induction coupling[C]//Electromagnetic Compatibility, Tokyo (EMC'14/Tokyo), 2014 International Symposium on IEEE. 2014: 449-452.

[9] Hirata A, Tsuchida S, Laakso I. Variability of SAR in different human models due to wireless power transfer with magnetic resonance. Electromagnetic Compatibility (EMC EUROPE), 2013 International Symposium on IEEE. 2013: 89-92.

[10] Christ A, Douglas M G, Roman J M, et al. Evaluation of wireless resonant power transfer systems with human electromagnetic exposure limits[J]. IEEE Transactions on Electromagnetic Compatibility. 2013; 55(2): 265-274.

[11] Minh Quoc Nguyen, Hughes Z, Woods P, et al. Field Distribution Models of Spiral Coil for Misalignment Analysis in wireless Power Transfer Systems[J]. IEEE Transactions on Microwave Theory and Techniques. 2014; 62(4): 920-930. 